\title{
Kraśkiewicz-Pragacz modules and some positivity properties of Schubert polynomials
}

\author{
Masaki Watanabe仰 \\ ${ }^{1}$ Graduate School of Mathematics, the University of Tokyo
}

\begin{abstract}
We use the modules introduced by Kraśkiewicz and Pragacz (1987, 2004) to show some positivity properties of Schubert polynomials. We give a new proof to the classical fact that the product of two Schubert polynomials is Schubert-positive, and also show a new result that the plethystic composition of a Schur function with a Schubert polynomial is Schubert-positive. The present submission is an extended abstract on these results and the full version of this work will be published elsewhere.
\end{abstract}

Résumé. Nous employons les modules introduits par Kraśkiewicz et Pragacz (1987, 2004) et démontrons certaines propriétés de positivité des polynômes de Schubert: nous donnons une nouvelle preuve pour le fait classique que le produit de deux polynômes de Schubert est Schubert-positif; nous d'emontrons aussi un nouveau résultat que la composition plethystique d'une fonction de Schur avec un polynôme de Schubert est Schubert-positif. Cet article est un sommaire de ces résultats, et une version pleine de ce travail sera publée ailleurs.

Keywords: Schubert polynomials, Schubert functors, Kraśkiewicz-Pragacz modules, Schubert calculus

\section{Introduction}

The study of Schubert polynomials is an important and interesting subject in algebraic combinatorics. One of the possible methods for studying Schubert polynomials is through the modules introduced by Kraśkiewicz and Pragacz ([KP87], [KP04]). Let $\mathfrak{b}$ be the Lie algebra of all upper triangular matrices. For each permutation $w$, Kraśkiewicz and Pragacz defined a representation $\mathcal{S}_{w}$ of $\mathfrak{b}$ such that its character with respect to the subalgebra $\mathfrak{h}$ of all diagonal matrices is equal to the Schubert polynomial $\mathfrak{S}_{w}$.

In [Wat14a], the author investigated characterizations of $\mathfrak{b}$-modules having a filtration whose subquotients are Kraśkiewicz-Pragacz modules (or KP filtration for short). The main motivation for this study is the investigation of positivity properties of Schubert polynomials. For example, it is a classical fact, usually proved using the cohomology ring of flag variety, that the product $\mathfrak{S}_{w} \mathfrak{S}_{v}$ of two Schubert polynomials is a positive sum of Schubert polynomials. If we show that the tensor product $\mathcal{S}_{w} \otimes \mathcal{S}_{v}$ of KP modules has a KP filtration, then it gives another proof to this fact. Another example is the question of positivity for the plethystic composition of a Schur function with a Schubert polynomial: if $\mathfrak{S}_{w}=x^{\alpha}+x^{\beta}+\cdots$ and $s_{\lambda}$ is a Schur function, is $s_{\lambda}\left(x^{\alpha}, x^{\beta}, \ldots\right)$ a positive sum of Schubert polynomials? A positive answer

\footnotetext{
$\dagger$ Email: mwata@ms.u-tokyo.ac.jp. 
to this question, a natural generalization of the Schur-positivity of the plethysm of two Schur functions, follows if we show the Schur-functor image $s_{\lambda}\left(\mathcal{S}_{w}\right)$ of a KP module has a KP filtration. In [Wat14b] we gave positive answers to both of these questions.

This extended abstract is a summary of the author's two papers [Wat14a] and [Wat14b]: the detailed arguments are given in these two papers and either omitted or only outlined here. The following sections are organized as follows. In Sections 2 and 3 we prepare some basic notations, and introduce the notion of Kraśkiewicz-Pragacz modules. In Section 4 we present some results from [Wat14a] giving a characterization of modules having a filtration by KP modules. In Section 5 we show some applications of our characterization: we present the result that the tensor product of KP modules has a KP filtration, and show that the plethystic composition of a Schur function with a Schubert polynomial is Schubert-positive.

\section{Preliminaries}

Let $\mathbb{N}$ be the set of all positive integers and let $\mathbb{Z}_{\geq 0}$ be the set of all nonnegative integers. A permutation $w$ is a bijection from $\mathbb{N}$ to itself which fixes all but finitely many points. Let $S_{\infty}$ denote the group of all permutations. Let $n$ be a positive integer. Let $S_{n}=\left\{w \in S_{\infty}: w(i)=i(i>n)\right\}$ and $S_{\infty}^{(n)}=\left\{w \in S_{\infty}: w(n+1)<w(n+2)<\cdots\right\}$. Sometimes a permutation will be written in one-line form: i.e. if $w \in S_{n}$, we may write $[w(1) w(2) \cdots w(n)]$ to mean $w$. For $i<j$, let $t_{i j}$ denote the permutation which exchanges $i$ and $j$ and fixes all other points. Let $s_{i}=t_{i, i+1}$. For a permutation $w$, let $\ell(w)=\#\{i<j: w(i)>w(j)\}$. Let $w_{0} \in S_{n}$ be the longest element of $S_{n}$, i.e. $w_{0}(i)=n+1-i(1 \leq i \leq n)$. For $w \in S_{\infty}^{(n)}$ we define $\operatorname{code}(w)=\left(\operatorname{code}(w)_{1}, \ldots, \operatorname{code}(w)_{n}\right) \in \mathbb{Z}_{\geq 0}^{n}$ by code $(w)_{i}=\#\{j: i<j, w(i)>w(j)\}$. We define the Rothe diagram $D(w)$ of a permutation $w$ as $D(w)=\{(i, w(j)): i<j, w(i)>w(j)\}$.

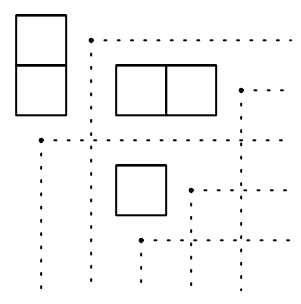

Figure 1: Rothe diagram of $w=$ [25143] (corresponding to the positions of the black dots above) is $D(w)=\{(1,1),(2,1),(2,3),(2,4),(4,3)\}$ (shown by the boxes above).

For a polynomial $f=f\left(x_{1}, \ldots, x_{n}\right)$ and $1 \leq i \leq n-1$, we define $\partial_{i} f=\frac{f-s_{i} f}{x_{i}-x_{i+1}}$. For $w \in S_{\infty}^{(n)}$ we can assign its Schubert polynomial $\mathfrak{S}_{w} \in \mathbb{Z}\left[x_{1}, \ldots, x_{n}\right]$, which is recursively defined by

- $\mathfrak{S}_{w}=x_{1}^{w(1)-1} x_{2}^{w(2)-1} \cdots x_{n}^{w(n)-1}$ if $w(1)>w(2)>\cdots>w(n)$, and

- $\mathfrak{S}_{w s_{i}}=\partial_{i} \mathfrak{S}_{w}$ if $\ell\left(w s_{i}\right)<\ell(w)$.

It is known that the set $\left\{\mathfrak{S}_{w}: w \in S_{\infty}^{(n)}\right\}$ constitutes a $\mathbb{Z}$-base for $\mathbb{Z}\left[x_{1}, \ldots, x_{n}\right]$ ([Mac91] (4.13)]).

Let $K$ be a field of characteristic zero. Let $\mathfrak{b}=\mathfrak{b}_{n}$ be the Lie algebra of all upper triangular $K$ matrices. and let $\mathfrak{h} \subset \mathfrak{b}$ and $\mathfrak{n}^{+} \subset \mathfrak{b}$ be the subalgebra of all diagonal matrices and the subalgebra of all strictly upper triangular matrices respectively. Let $\mathcal{U}(\mathfrak{b})$ and $\mathcal{U}\left(\mathfrak{n}^{+}\right)$be the universal enveloping algebras 
of $\mathfrak{b}$ and $\mathfrak{n}^{+}$respectively. For a $\mathcal{U}(\mathfrak{b})$-module $M$ and $\lambda=\left(\lambda_{1}, \ldots, \lambda_{n}\right) \in \mathbb{Z}^{n}$, let $M_{\lambda}=\{m \in M$ : $h m=\langle\lambda, h\rangle m(\forall h \in \mathfrak{h})\}$ where $\langle\lambda, h\rangle=\sum \lambda_{i} h_{i}$. If $M_{\lambda} \neq 0$ then $\lambda$ is said to be a weight of $M$. If $M=\bigoplus_{\lambda \in \mathbb{Z}^{n}} M_{\lambda}$ and each $M_{\lambda}$ has a finite dimension, then $M$ is said to be a weight $\mathfrak{b}$-module and we define $\operatorname{ch}(M)=\sum_{\lambda} \operatorname{dim} M_{\lambda} x^{\lambda}$ where $x^{\lambda}=x_{1}^{\lambda_{1}} \cdots x_{n}^{\lambda_{n}}$. From here we only consider weight $\mathfrak{b}$-modules, and write $\operatorname{Ext}^{i}$ to mean Ext functors in the category of all weight $\mathfrak{b}$-modules. For $1 \leq i<j \leq n$, let $e_{i j} \in \mathfrak{b}$ be the matrix with 1 at the $(i, j)$-position and all other coordinates 0 . For $\lambda \in \mathbb{Z}^{n}$, let $K_{\lambda}$ denote the one-dimensional $\mathcal{U}(\mathfrak{b})$-module where $h \in \mathfrak{h}$ acts by $\langle\lambda, h\rangle$ and $e_{i j}$ acts by 0 . We define $\rho=(n-1, n-2, \ldots, 0) \in \mathbb{Z}^{n}, \mathbf{1}=(1, \ldots, 1) \in \mathbb{Z}^{n}$ and $\epsilon_{j}=(0, \ldots, 1, \ldots, 0) \in \mathbb{Z}^{n}$ (1 at $j$-th position).

\section{Kraśkiewicz-Pragacz modules}

In [KP87], Kraśkiewicz and Pragacz defined certain $\mathcal{U}(\mathfrak{b})$-modules which we call here KraśkiewiczPragacz modules or KP modules. Here we use the following definition. Let $w \in S_{\infty}^{(n)}$. Let $K^{n}=$ $\bigoplus_{1 \leq i \leq n} K u_{i}$ be the vector representation of $\mathfrak{b}$. For each $p \in \mathbb{N}$, let $\{i:(i, p) \in D(w)\}=\left\{i_{1}, \ldots, i_{l_{p}}\right\}$ $\left(i_{1}<\cdots<i_{l_{p}}\right)$, and let $u_{w}^{(p)}=u_{i_{1}} \wedge \cdots \wedge u_{i_{l_{p}}}$. Let $u_{w}=u_{w}^{(1)} \otimes u_{w}^{(2)} \otimes \cdots \in \bigwedge^{l_{1}} K^{n} \otimes \bigwedge^{l_{2}} K^{n} \otimes \cdots$. Then the KP module $\mathcal{S}_{w}$ associated to $w$ is defined as $\mathcal{S}_{w}=\mathcal{U}(\mathfrak{b}) u_{w}$. This module satisfies the following:

Theorem 3.1 ([KP04] Remark 1.6 and Theorem 4.1]) $\mathcal{S}_{w}$ is a weight module and $\operatorname{ch}\left(\mathcal{S}_{w}\right)=\mathfrak{S}_{w}$.

Example 3.2 If $w=s_{i}$, then $D\left(s_{i}\right)=\{(i, i)\}, u_{s_{i}}=u_{i}$ and $\mathcal{S}_{s_{i}}=\bigoplus_{1 \leq j \leq i} K u_{j}=$ : $K^{i} . \operatorname{So} \operatorname{ch}\left(\mathcal{S}_{s_{i}}\right)=$ $x_{1}+\cdots+x_{i}=\mathfrak{S}_{s_{i}}$.

Example 3.3 More generally, if $w \in S_{\infty}^{(n)}$ is a Grassmannian permutation with descent at $i$-th position, i.e. $w(1)<\cdots<w(i)$ and $w(i+1)<w(i+2)<\cdots$, then the corresponding Rothe diagram is, after a rearrangement of the columns, a Young diagram in French notation (see Figure 2). In this case $u_{w}$ is a lowest-weight vector in a irreducible representation of $\mathfrak{g l}_{i}$ with lowest weight $\operatorname{code}(w)$, and thus $\mathcal{S}_{w}$ is isomorphic to this module (seen as a module over $\mathfrak{b}_{n}$ through the morphism $\mathfrak{b}_{n} \ni e_{p q} \mapsto\left\{\begin{array}{ll}e_{p q} & (q \leq i) \\ 0 & (q>i)\end{array} \in \mathfrak{b}_{i} \hookrightarrow \mathfrak{g l}_{i}\right)$. This corresponds to the fact that Schubert polynomials indexed by permutations are just the Schur polynomials.

Example 3.4 If $w$ is 2143-avoiding, then it is known ([Mac91. (1.27)]) that the rows of D(w) are totally (pre)ordered by inclusion. Thus in this case $u_{w}$ is an extremal weight vector in a certain irreducible representation of $\mathfrak{g l}_{n}$, and thus $\mathcal{S}_{w}$ is isomorphic to a Demazure module: i.e. the $\mathcal{U}(\mathfrak{b})$-module generated by an extremal weight vector in a irreducible representation of $\mathfrak{g l}_{n}$. Note that this corresponds to the result of Lascoux and Schutzenberger ([LS89]. Theorem 5], [Las03. Corollary 10.5.2]) that Schubert polynomials with 2143-avoiding indices are equal to certain key polynomials.

On the other hand, consider $w=[2143]$. Then $D(w)=\{(1,1),(3,3)\}, u_{w}=u_{1} \otimes u_{3}, \mathcal{S}_{w}=$ $\bigoplus_{1 \leq i \leq 3} K\left(u_{1} \otimes u_{i}\right)=K^{1} \otimes K^{3}$ and $\operatorname{ch}\left(\mathcal{S}_{w}\right)=x_{1}\left(x_{1}+x_{2}+x_{3}\right)=\mathfrak{S}_{w}$. In this case $\mathcal{S}_{w}$ is not isomorphic to the Demazure module of the same lowest weight ${ }^{(i)}$ In general, $\mathcal{S}_{w}$ is isomorphic to the

(i) The KP module $\mathcal{S}_{[2143]}$ in this example is, if not seen as a $\mathcal{U}(\mathfrak{b})$-module but as a $\mathcal{U}\left(\mathfrak{n}^{+}\right)$-module, isomorphic to a Demazure module (say $V(0,0,1)$ ), and thus results such as Theorem 4.3 for this kind of KP modules follow from known results on Demazure 
Demazure module $V(\operatorname{code}(w))$ of lowest weight $\operatorname{code}(w)$ if and only if $w$ is 2143-avoiding. For a general $w$, there always exists a surjection from $\mathcal{S}_{w}$ to $V(\operatorname{code}(w))$.

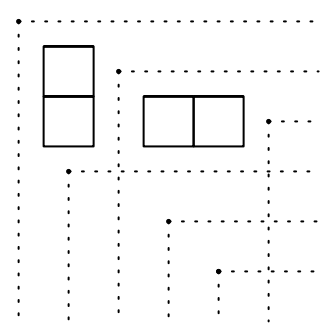

Figure 2: Rothe diagram of a Grassmannian permutation $w=[136245]$. Here $u_{w}=\left(u_{2} \wedge u_{3}\right) \otimes u_{3} \otimes u_{3}$ is a lowest vector in an irreducible representation of $\mathfrak{g l}_{3}$ with lowest weight $(0,1,3)$.

For convenience, we slightly generalize the notion of Schubert polynomials and KP modules. For $\lambda=\operatorname{code}(w) \in \mathbb{Z}_{\geq 0}^{n}\left(w \in S_{\infty}^{(n)}\right)$ we write $\mathfrak{S}_{\lambda}=\mathfrak{S}_{w}$ and $\mathcal{S}_{\lambda}=\mathcal{S}_{w}$. For a general $\lambda \in \mathbb{Z}^{n}$ take $k \in \mathbb{Z}$ so that $\lambda+k \mathbf{1} \in \mathbb{Z}_{>0}^{n}$ and define $\mathfrak{S}_{\lambda}=x^{-k 1} \mathfrak{S}_{\lambda+k \mathbf{1}}$ and $\mathcal{S}_{\lambda}=K_{-k \mathbf{1}} \otimes \mathcal{S}_{\lambda+k \mathbf{1}}$ (note that this does not depend on the choice of $k$ ). We use both permutations and integer vectors as indices of Schubert polynomials and KP modules, but we believe that it will cause no confusion.

A $K P$ filtration of a weight $\mathfrak{b}$-module $M$ is a filtration $0=M_{0} \subset M_{1} \subset \cdots \subset M_{r}=M$ of $\mathfrak{b}$-modules such that each $M_{i} / M_{i-1}$ is isomorphic to some $\mathcal{S}_{\lambda^{i}}\left(\lambda^{i} \in \mathbb{Z}^{n}\right)$. It is clear from the definition that if $M$ has a KP filtration then $\operatorname{ch}(M)$ is a positive sum of Schubert polynomials.

\section{A criterion for the existence of KP filtration}

In [Wat14a], the author investigated characterizations of modules having KP filtrations. The method we used there is similar to the method used in the study of Demazude modules and excellent filtrations ([Pol89], [vdK89]): the use of the theory of highest-weight categories ([CPS88]).

In order to develop an analogue of the theory on excellent filtration for KP modules using the highestweight category theory, an important step is to characterize KP modules as projective objects in certain categories of $\mathfrak{b}$-modules, as Polo did for Demazure modules in [Pol89]. We proved in [Wat14a] the following such characterization.

Introduce an order $\leq$ on $\mathbb{Z}^{n}$ as follows. For $\lambda, \mu \in \mathbb{Z}^{n}$, take $k$ so that $\lambda+k \mathbf{1}, \mu+k \mathbf{1} \in \mathbb{Z}_{\geq 0}^{n}$, and define $\lambda \leq \mu \Longleftrightarrow \ell(w)=\ell(v)$ and $w^{-1} \underset{\operatorname{lex}}{\geq v^{-1}}$ where $w, v \in S_{\infty}^{(n)}$ are defined by $\operatorname{code}(w)=\lambda+k \mathbf{1}$ and $\operatorname{code}(v)=\mu+k \mathbf{1}$. Here $\underset{\text { lex }}{\geq}$ denotes the lexicographic ordering on permutations. For $\lambda \in \mathbb{Z}_{\geq 0}^{n}$, let $\mathcal{C}_{\leq \lambda}$ be the category of all weight $\mathfrak{b}$-modules whose weights are all $\leq \lambda$.

Theorem 4.1 ([Wat14a, Proposition 6.4]) The modules $\mathcal{S}_{\lambda}$ and $\mathcal{S}_{\rho-\lambda}^{*} \otimes K_{\rho}$ are in $\mathcal{C}_{\leq \lambda}$ and give the projective cover and the injective hull of $K_{\lambda}$ in $\mathcal{C}_{\leq \lambda}$ respectively.

Example 4.2 Let $n=3$. Then the weights $\lambda \in \mathbb{Z}^{n}$ which are less than or equal to $(0,2,1)$, with respect to the ordering $\leq$, are $(1,1,1),(2,1,0),(1,2,0),(2,0,1),(3,0,0),(1,0,2)$ and $(0,2,1)$. Then it is easy to

modules. But in fact there also exist KP modules which are, even as a $\mathcal{U}\left(\mathfrak{n}^{+}\right)$-module, not isomorphic to any Demazure modules: an example is $\mathcal{S}_{[13254]} \cong K^{2} \otimes K^{4}$. 
check that the projective cover of $K_{(0,2,1)}$ in $\mathcal{C}_{\leq(0,2,1)}$ is isomorphic to the KP module $\mathcal{S}_{(0,2,1)}=\mathcal{S}_{[1432]}$, which has character $\mathfrak{S}_{[1432]}=x_{1}^{2} x_{2}+x_{1}^{2} x_{3}+x_{1} x_{2}^{2}+x_{1} x_{2} x_{3}+x_{2}^{2} x_{3}$.

In the proof of this characterization, we used the following structural result on KP modules. The current proof of the following result in [Wat14a] uses a long case-by-case check, and we hope that there is a more elegant proof.

For $w \in S_{\infty}^{(n)}$ and $1 \leq i<j \leq n$, let $C_{i j}(w)=\{p:(i, p) \notin D(w),(j, p) \in D(w)\}(=\{w(k)$ : $k>j, w(i)<w(k)<w(j)\})$ and let $m_{i j}(w)=\left|C_{i j}(w)\right|$. Then, since $e_{i j}^{2} u_{w}^{(p)}=0$ for $p \in C_{i j}(w)$ and $e_{i j} u_{w}^{(p)}=0$ for $p \notin C_{i j}(w)$, we see that $e_{i j}^{m_{i j}(w)+1}$ annihilates $u_{w}=u_{w}^{(1)} \otimes u_{w}^{(2)} \otimes \cdots$. The following theorem states that the annihilator of $u_{w}$ is in fact generated by these elements:

Theorem 4.3 ([Wat14a, Theorem 4.1]) Let $I_{w}$ is the left ideal of $\mathcal{U}\left(\mathfrak{n}^{+}\right)$generated by the elements $e_{i j}^{m_{i j}(w)+1}(1 \leq i<j \leq n)$. Then there exists an $\mathcal{U}\left(\mathfrak{n}^{+}\right)$-module isomorphism $\mathcal{U}\left(\mathfrak{n}^{+}\right) / I_{w} \cong \mathcal{S}_{w}$ sending $1 \bmod I_{w}$ to $u_{w}$.

Example 4.4 Let $n=4$ and $w=[25143] \in S_{\infty}^{(4)}$. Then the Rothe diagram $D(w)$ is as in Figure 1, and $I_{w}$ is generated by $e_{12}^{3}, e_{13}, e_{14}^{2}, e_{23}, e_{24}$ and $e_{34}^{2}$.

Note that Theorem 4.3 can be seen as an analog of the Joseph's result ([Jos85, Theorem 3.4]) on Demazure modules which states (in $\mathfrak{g l}_{n}$ case) that the annihilator ideal of the lowest weight vector of weight $\lambda \in \mathbb{Z}^{n}$ in a Demazure module is generated by $e_{i j}^{\max \left\{\lambda_{j}-\lambda_{i}, 0\right\}+1}(1 \leq i<j \leq n)$.

Using Theorem 4.1, the following characterization of modules having KP filtrations can be shown with a standard argument from the theory of highest-weight categories (in fact, one more trick is needed [Wat14a, Lemma 7.1]):

Theorem 4.5 ([Wat14a, Corollary 7.6, Theorem 8.1]) Let $M$ be a finite-dimensional weight $\mathfrak{b}$-module. Then the followings are equivalent:

1. M has a KP filtration.

2. $\operatorname{Ext}^{i}\left(M, \mathcal{S}_{\lambda}^{*} \otimes K_{\rho}\right)=0$ for all $i \geq 1$ and $\lambda \in \mathbb{Z}^{n}$.

3. $\operatorname{Ext}^{1}\left(M, \mathcal{S}_{\lambda}^{*} \otimes K_{\rho}\right)=0$ for all $\lambda \in \mathbb{Z}^{n}$.

\section{Applications}

In this section we present two applications of Theorem 4.5. One application is the proof of the fact that the tensor product $\mathcal{S}_{w} \otimes \mathcal{S}_{v}$ of KP modules admits a KP filtration. This can be seen as a module-theoretic counterpart of the classical fact that the product $\mathfrak{S}_{w} \mathfrak{S}_{v}$ of Schubert polynomials is a positive sum of Schubert polynomials, and it also gives a new proof to this fact. Another application is the Schubertpositivity of the "plethystic composition" $s_{\lambda}\left[\mathfrak{S}_{w}\right]$ of a Schur function with a Schubert polynomial.

First we prepare some useful corollaries from Theorem 4.5 .

Proposition 5.1 1. If $M=M_{1} \oplus \cdots \oplus M_{r}$, then $M$ has a KP filtration if and only if each $M_{i}$ has.

2. If $0 \rightarrow L \rightarrow M \rightarrow N \rightarrow 0$ is exact and $M$ and $N$ have KP filtrations, then $L$ also has a KP filtration. 
Proof: 1. is easy since $\operatorname{Ext}^{1}(M, N)=\bigoplus \operatorname{Ext}^{1}\left(M_{i}, N\right)$ for any $N$. 2. also follows from Theorem 4.5 since if $0 \rightarrow L \rightarrow M \rightarrow N \rightarrow 0$ is an exact sequence then we have an exact sequence $\operatorname{Ext}^{1}(M, A) \rightarrow$ $\operatorname{Ext}^{1}(L, A) \rightarrow \operatorname{Ext}^{2}(N, A)$ for any $A$.

Using this, we can show that the tensor product of KP modules admits a KP filtration:

Theorem 5.2 ([Wat14b, Theorem 4.1]) For any $w, v \in S_{\infty}^{(n)}$, the module $\mathcal{S}_{w} \otimes \mathcal{S}_{v}$ have a KP filtration.

Outline of Proof: First check the case $w=s_{i}$ (this corresponds to Monk's formula for Schubert polynomials) by explicitly constructing a filtration. Then we see that $\mathcal{S}_{s_{i}} \otimes \mathcal{S}_{s_{j}} \otimes \cdots \otimes \mathcal{S}_{s_{k}} \otimes \mathcal{S}_{v}$ have a KP filtration for any $i, j, \ldots, k$ and $v \in S_{\infty}^{(n)}$, and by Proposition 5.1 $1, \bigwedge^{j_{1}} \mathcal{S}_{s_{i_{1}}} \otimes \cdots \otimes \bigwedge^{j_{r}} \mathcal{S}_{s_{i_{r}}} \otimes \mathcal{S}_{v}$ have a KP filtration for any $i_{1}, j_{1}, \ldots, i_{r}, j_{r}$ and $v \in S_{\infty}^{(n)}$.

If $w \in S_{n}$, it can be shown, also using the cases of Monk's formula, that for suitable $i_{1} \ldots, i_{n-1}$ there exists an exact sequence $0 \rightarrow \mathcal{S}_{w} \rightarrow \bigwedge^{i_{1}} \mathcal{S}_{s_{1}} \otimes \cdots \otimes \bigwedge^{i_{n-1}} \mathcal{S}_{s_{n-1}} \rightarrow N \rightarrow 0$ such that $N$ have a KP filtration whose subquotients are of the form $\mathcal{S}_{u}\left(u^{-1}>w^{-1}\right)$. Then the claim follows by the induction on $w$ using the exact sequence $0 \rightarrow \mathcal{S}_{w} \otimes \mathcal{S}_{v} \rightarrow \bigwedge^{i_{1}} \mathcal{S}_{s_{1}} \otimes \cdots \otimes \bigwedge^{i_{n-1}} \mathcal{S}_{s_{n-1}} \otimes \mathcal{S}_{v} \rightarrow N \otimes \mathcal{S}_{v} \rightarrow 0$ and Proposition 5.12.

For a general $w$ the claim follows by letting $n$ large, since for $n^{\prime}>n, \mathfrak{b}_{n}$-modules can be naturally identified with $\mathfrak{b}_{n^{\prime}}$-modules on which $e_{i j}(j>n)$ acts by zero and under this identification the $\mathfrak{b}_{n}$-module $\mathcal{S}_{w}\left(w \in S_{\infty}^{(n)} \subset S_{\infty}^{\left(n^{\prime}\right)}\right)$ corresponds to the $\mathfrak{b}_{n^{\prime}}$-module $\mathcal{S}_{w}$.

Remark 5.3 As we pointed in [Wat14a] and [Wat14b], Theorem 5.2 gives a new description of generalized Littlewood-Richardson coefficients, in a manner somewhat similar to triple-intersection formula in the geometric theory of Schubert calculus: for $u, v, w \in S_{n}$, if the polynomial $\mathfrak{S}_{u} \mathfrak{S}_{v}$ is expanded into a sum of Schubert polynomials, the coefficient of $\mathfrak{S}_{w}$ is equal to the dimension of $\operatorname{Hom}_{\mathfrak{b}}\left(\mathcal{S}_{u} \otimes \mathcal{S}_{v}, \mathcal{S}_{w_{0} w}^{*} \otimes\right.$ $\left.K_{\rho}\right) \cong \operatorname{Hom}_{\mathfrak{b}}\left(\mathcal{S}_{u} \otimes \mathcal{S}_{v} \otimes \mathcal{S}_{w_{0} w}, K_{\rho}\right)$.

As a corollary of Theorem 5.2 we can show that the plethystic composition of a Schur function with a Schubert polynomial is Schubert-positive:

Corollary 5.4 (|Wat14b , Corollary 4.3]) Let $\lambda$ be a partition and $s_{\lambda}$ be the corresponding Schur function. Let $w \in S_{\infty}^{(n)}$ and write $\mathfrak{S}_{w}$ as a sum of monomials as $\mathfrak{S}_{w}=x^{\alpha}+x^{\beta}+\cdots$. Then $s_{\lambda}\left[\mathfrak{S}_{w}\right]:=$ $s_{\lambda}\left(x^{\alpha}, x^{\beta}, \ldots\right)$ is a positive sum of Schubert polynomials.

Proof: Let $s_{\lambda}$ denote the Schur functor corresponding to $\lambda$ (here we are using the same symbol as Schur functions, but we hope that it would cause no confusion). Then $s_{\lambda}\left(\mathcal{S}_{w}\right)$ is a direct sum component of $\mathcal{S}_{w}^{\otimes|\lambda|}$, and thus, by Proposition 5.11 and Theorem 5.2 have a KP filtration. Thus the claim follows since $s_{\lambda}\left[\mathfrak{S}_{w}\right]$ is the character of $s_{\lambda}\left(\mathcal{S}_{w}\right)$.

Remark 5.5 The method we used here, i.e. the use of KP modules and highest-weight category theory for the study of Schubert positivity, is of course not only applicable for the proof of the positivity of product and plethystic composition but also for the investigation of other positivity properties of Schubert polynomials. For example, by considering the restriction of KP modules to certain subalgebra of $\mathfrak{b}$ and using the similar method here (i.e. using highest-weight category theory to reduce the problem to the simplest case $\left.\mathfrak{S}_{s_{i}}\right)$, it can be shown that for any $w \in S_{\infty}^{(n)}$ and $I=\left\{i_{1}<\cdots<i_{r}\right\}, J=\left\{j_{1}<\cdots<j_{s}\right\}$ 
with $I \sqcup J=\{1, \ldots, n\}$, the polynomial $\mathfrak{S}_{w}\left(z_{1}, \ldots, z_{n}\right)\left(z_{k}=\left\{\begin{array}{ll}x_{p} & \left(k=i_{p}\right) \\ y_{q} & \left(k=j_{q}\right)\end{array}\right)\right.$ is a positive sum of polynomials of the form $\mathfrak{S}_{u}\left(x_{1}, \ldots, x_{r}\right) \mathfrak{S}_{v}\left(y_{1}, \ldots, y_{s}\right)$. As one of the referees pointed out, this result appears in a work [BS98. Theorem 4.5.4] of Bergeron and Sottile, where the coefficient appearing in the restriction is related to the multiplication coefficients. It would be an interesting problem to investigate module-theoretic counterpart to this relation.

\section{References}

[BS98] N. Bergeron and F. Sottile. Schubert polynomials, the Bruhat order, and the geometry of flag manifolds. Duke Math. J., 95(2):373-423, 1998.

[CPS88] E. Cline, B. Parshall, and L. Scott. Finite-dimensional algebras and highest weight categories. J. Reine Angew. Math., 391:85-99, 1988.

[Jos85] A. Joseph. On the Demazure character formula. Ann. Sci. École Norm. Sup. (4), 18(3):389-419, 1985.

[KP87] W. Kraśkiewicz and P. Pragacz. Foncteurs de Schubert. C. R. Acad. Sci. Paris Sér. I Math., 304(9):209-211, 1987.

[KP04] W. Kraśkiewicz and P. Pragacz. Schubert functors and Schubert polynomials. Eur. J. Comb., 25(8):1327-1344, 2004.

[LS89] A. Lascoux and M.-P. Sch'utzenberger. Tableaux and noncommutative Schubert polynomials. Funct. Anal. Appl., 23:63-64, 1989.

[Las03] A. Lascoux. Symmetric Functions and Combinatorial Operators on Polynomials. C.B.M.S. Reg. Conf. Ser. Maths. 99, 2003.

[Mac91] I. G. Macdonald. Notes on Schubert Polynomials. LACIM, Université du Québec à Montréal, 1991.

[Po189] P. Polo. Variétés de Schubert et excellentes filtrations. Astérisque, (173-174):10-11, 281-311, 1989. Orbites unipotentes et représentations, III.

[vdK89] W. van der Kallen. Longest weight vectors and excellent filtrations. Math. Z., 201(1):19-31, 1989.

[vdK93] W. van der Kallen. Lectures on Frobenius Splittings and B-modules. Springer, 1993.

[Wat14a] M. Watanabe. Filtrations of $\mathfrak{b}$-modules with successive quotients isomorphic to Kraśkiewicz and Pragacz's modules realizing Schubert polynomials as their characters. preprint, arXiv: $1406.6203 v 3$, June 2014.

[Wat14b] M. Watanabe. Tensor product of Kraśkiewicz and Pragacz's modules. preprint, arXiv:1410.7981v1, October 2014. 
